\title{
ISBER Best Practices Fourth Edition: A Success Story
}

\author{
Zisis Kozlakidis, ${ }^{1,2}$ Catherine Seiler, ${ }^{3,4}$ and Daniel Simeon-Dubach ${ }^{5,6}$
}

$\mathrm{T}$ He ISBER Best Practices Fourth edition is a true success story and will continue to impact biobanking collection and discovery even more in the future. After an intensive and busy revision and extension of the previous version by a very committed editorial board and with the input of numerous contributions of many ISBER members from across the world, the fourth edition was released in February 2018 and can now be downloaded for free from the ISBER website (www.isber.org/bestpractices).

To prepare for a successful launch, members of different ISBER committees and the ISBER Head Office joined forces. Under the leadership of the Education \& Training (E\&T) Committee and with members from the Communication, Science Policy, and Standards Committees, a webinar was organized focusing on the background and reasons for updating the Best Practices and presenting the changes to the new edition. This webinar took place 1 day after the release and was very well received with $>80$ attendees. In parallel, the Communication Committee along with the ISBER Head Office prepared press releases and communication material that was sent to other scientific and biobanking organizations to help spread the news. At the ISBER 2018 Biospecimen Research Symposium in February in Luxembourg, several posters on the ISBER Best Practices were presented. Promotional activities for the Best Practices continue far past launch, including posters and two workshops, one lead by E\&T, at the ISBER 2018 Annual Meeting \& Exhibits in Dallas, TX. More is planned for several meetings and events throughout the rest of the year as well.

In support of the Best Practice's international reach, since the release of the fourth edition, a large group of engaged people from different countries started the immense task of translating the English version of the ISBER Best Practices fourth edition into their own language. This initial translation effort has focused on Chinese, Japanese, Korean, French, and Spanish. The Chinese translation was available on March 30 at the 10th anniversary BBCMBA (Biobank Branch, China Medicinal Biotech Association) conference in Shanghai where several hundred copies were distributed among the participants. As the additional translations are released, they will be made available on the ISBER website (www.isber .org/bestpractices).

What have we achieved with the Best Practices thus far? As of the end of April, almost 900 people have downloaded the ISBER Best Practices. We have had the following organizations comment and promote the release of the Best Practices: BBMRI-ERIC, ATCC, SmarterLab, BiKE_Biobanking, Pointlab, GGBN, and the Society for Cryobiology. Importantly, a large number of individuals downloading the Best Practices stated that their intended use is for the development of SOPs and/or for informing local policy guidelines. This is evidence of the impact the Best Practices continue to have on biobanking operations globally.

We expect a significant boost in download during the ISBER 2018 Annual Meeting and when the different translations become available. In addition, tools and programs that are based on the ISBER Best Practices will be updated in the near future to include the changes from the fourth edition. These tools include the ISBER Self-Assessment Tool and SPREC (Standard PREanalytical Code), but also other programs such as the College of American Pathologists Biorepository Accreditation Program, which are, in part, based on the ISBER Best Practices. These tools and programs are a crucial part of improving professionalism in biobanking.

The ISBER Best Practices fourth edition is not simply an update on previous versions. Significant efforts have been made to widen the scope of the Best Practices, reflecting a growing scientific field in addition to providing an extensive international scope. The ISBER Best Practices are intended to be used as a global reference manual for the creation and operation of biobanks at the highest standard, to facilitate the acquisition and sharing of research-ready biosamples, and data to support a reliable and reproducible scientific pursuit. The Best Practices truly encourage users to consider and optimize the journey "From Collection to Discovery." They are resource agnostic, equally addressing high- and

\footnotetext{
${ }^{1}$ Division of Infection and Immunity, University College London, London, United Kingdom.

${ }^{2}$ ISBER President.

${ }^{3}$ Kaleido Biosciences, Bedford, Massachusetts.

${ }^{4}$ Chair, ISBER Communication Committee.

${ }^{5}$ Medservice, Biobanking Consulting and Services, Walchwil, Switzerland.

${ }^{6}$ Chair, ISBER Standards Committee.
} 
low-resource availability settings, including medical, environmental, disease-oriented, and population-based biobanks. The ISBER Best Practices constitute guidelines that, if applied appropriately and consistently, should allow organizations to obtain local and national standards and certifications, and will be the first step toward preparing biobanks for applying international standards, such as the upcoming ISO standard for biobanking.

ISBER, as the only global society of biobanking professionals from both the public and private sectors, is committed to advancing the field of biobanking by regularly updating the Best Practices for biobanking (first ed. 2005, second ed. 2008, third ed. 2012, and fourth ed. 2018). The ISBER Best Practices have been applied in practice many times over the past 20 years and have now been recognized as the premiere handbook globally in establishing up and operating a biobank, referenced as a cornerstone of operations a few hundred times in international peer-reviewed academic literature.

The biobanking community is equipped to develop and grow further based on the firm foundations set by the ISBER Best Practices. However, the true test of the fourth edition's utility will be through its implementation. Depending on the local situation (type of biobank, what types of services the biobank provides, availability of resources, etc.), this implementation can be handled in different ways. Some biobanks might be able and capable to fully implement the Best Practices. Others will select those sections or part of it that make the most sense at the time. However, this selection should be done after an educated and in-depth analysis of the specific situation the biobank is in.

As biobanking science is progressing rapidly, a dedicated task force has already been created to start planning for the fifth edition of the ISBER Best Practices. This task force will develop strategies for how to best organize this update and make the document as user friendly as possible. Once vetted by the ISBER Board of Directors, we will inform the ISBER community of how the monumental task of creating the fifth edition will proceed. We will also engage the IBSER community - this growing community of global biobanking experts - to contribute to the next update. ISBER as an organization is not only listening to its members, but also an organization providing them with the tools and evidence-based practices to continue developing and expanding the biobanking field globally.

Address correspondence to: Zisis Kozlakidis, MBA, PhD Division of Infection and Immunity University College London The Farr Institute of Health Informatics Research 222 Euston Road NW1 2DA, London United Kingdom

E-mail: z.kozlakidis@ucl.ac.uk 